\title{
Atención socioeducativa y trastorno mental severo: la vivienda como base de intervención
}

\author{
Socio-educational care and severe mental disorder: \\ housing as a basis for intervention
}

\author{
Omar García-Pérez / garciaomar@uniovi.es \\ José Vicente Peña-Calvo / vipe@uniovi.es \\ Susana Torío-López / storio@uniovi.es \\ Universidad de Oviedo, España
}

\begin{abstract}
The paradigm shift in mental health opens the door to a multidisciplinary approach. We advocate the need to invest in the recovery of people with Severe Mental Disorder from a socio-educational perspective, beyond the classic medical-clinical approach. The literature is reviewed and the results of various programs to support housing as a centerpiece of community intervention are analyzed. Improved personal and social functioning, fewer revenues, greater satisfaction and quality of life at lower cost is evidence. It is therefore crucial setting public policy of social action that promotes the conditions necessary to achieve social justice and inclusive citizenship. The relevance of Social Pedagogy and Social Education in achieving this goal and in improving their quality of life is concluded. We demand their theoretical and practical space in the field of mental health.
\end{abstract}

Key words: mental illness, housing, social inclusion, socio-educational intervention, social justice.

Resumen: El cambio de modelo de atención en salud mental abre las puertas a un abordaje multiprofesional. Defendemos la necesidad de apostar por la recuperación de las personas con trastorno mental severo desde una vertiente socioeducativa, más allá del planteamiento médico-clínico. Se revisa la bibliografía y analizan los resultados de diversos programas de apoyo a la vivienda como eje central de intervención en la comunidad. Se evidencia un mejor funcionamiento personal y social, menor número de ingresos, mayor satisfacción y calidad de vida a menor coste económico. Es crucial la configuración de una política pública de acción social que promueva las condiciones necesarias para conseguir una justicia social y una ciudadanía inclusiva. Se concluye la relevancia de la Pedagogía Social y la Educación Social en la consecución de este objetivo y en la mejora de la calidad de vida. Reivindicamos su propio espacio teórico-práctico en el ámbito de salud mental.

Palabras clave: enfermedad mental, vivienda, integración social, intervención socioeducativa, justicia social. 


\section{Introducción}

Actualmente, la consideración de "ciudadano" que se le otorga a la persona con enfermedad mental provoca un cambio en la forma de atender sus necesidades, abriendo el abanico de posibilidades a una atención multidisciplinar. Esta concepción, más filosófica que práctica real, ha guiado la propuesta de transformación de la atención psiquiátrica en la última mitad del siglo pasado, aunque con diferentes variantes, en Estados Unidos, diversos países de Europa y América Latina. En este paradigma, la dimensión pedagógica ha de convertirse en un elemento indispensable para la inclusión social de las personas con trastorno mental severo (TMS) en la comunidad.

En ese proceso, desde nuestra visión, la prestación de servicios residenciales y de alojamiento con apoyo emerge como uno de los pilares básicos en la recuperación e inclusión social de las personas con enfermedad mental, hasta el punto de que varios autores, como Shepherd y Murray (citados en Macpherson et al., 2004: 180), sostienen que "la vivienda debe estar en el centro de la psiquiatría comunitaria”.

A continuación se presentan algunas cuestiones socioeducativas clave que suscitan la reflexión sobre la atención al TMS y los programas de vivienda. En primer lugar, se destaca la importancia que supuso el cambio de modelo de atención en salud mental en España, pasando de una concepción puramente terapéutica y clínica bajo un modelo institucional, a otra visión holística, comunitaria y ciudadana, abriendo las puertas a un abordaje intersectorial (Prieto-Rodríguez, 2002).

Asimismo, se da cuenta de las necesidades de las personas con TMS y de los diferentes ámbitos de intervención pedagógica; finalmente, se considera a la vivienda como eje central de dicha intervención, debido a los efectos positivos que produce en sus usuarios, proporcionando los apoyos individualizados necesarios en función de las múltiples demandas personales y sociales de sus residentes. Por tanto, el objetivo del que parte este trabajo es mostrar la relevancia del trabajo socioeducativo en la labor de recuperación de las personas con TMS, así como su importancia en las políticas sociales que debieran hacerla efectiva. Sin esa visión socioeducativa, desde todos los niveles administrativos, políticos y laborales, la pretendida integración social queda incompleta, reivindicando una política basada en la justicia social. 


\section{Atención al trastorno mental severo en España: ¿ una cuestión pedagógica?}

Históricamente, el concepto de trastorno mental ha experimentado varios giros significativos de los que se derivan claras implicaciones en términos de políticas y estrategias de atención, que han exigido y exigen en la actualidad cambios organizativos de gran calado. Hasta bien entrado el siglo XIX, la locura era considerada una problemática social y una cuestión de orden público, cuyo tratamiento era la reclusión (Foucault, 1976). Posteriormente, la locura pasó a ser considerada una enfermedad y, como tal, un asunto médico, transformando los asilos en hospitales psiquiátricos. Sin embargo, la función custodial seguía primando sobre el tratamiento terapéutico (Rodríguez, 2002).

A lo largo del siglo XX surgen voces críticas hacia el modelo institucional y reclaman una transformación. Incluso, el origen y causa de las enfermedades mentales es discutido, generando diversas corrientes de pensamiento que repercuten en la forma de proceder ante tales padecimientos. Por un lado, nos encontramos con un modelo biomédico hegemónico, en el cual el diagnóstico psiquiátrico es el resultado de un juicio clínico basado en un enfoque cerebral de la enfermedad mental, con una etiología biológica y cuyas técnicas de tratamiento son de carácter farmacológico (Cea-Madrid, 2015; Geneyro y Tirado, 2015).

Por otro lado, se sitúa la corriente derivada de la denominada antipsiquiatría de los años sesenta y setenta, en la que autores como Cooper, Laing, Basaglia, Oury o Szasz, plantean la hipótesis del origen social de la enfermedad mental, de modo que van a caracterizar los trastornos psicóticos, más concretamente la esquizofrenia, como un problema relacional y no orgánico; es decir, como un trastorno derivado de la adaptación del sujeto a su entorno social (Cea-Madrid y Castillo-Parada, 2016; Desviat, 2006; Morales, 2012; Pastor y Ovejero, 2009), hasta el punto de negar la existencia de un trastorno mental y usar la enfermedad mental como un mecanismo social, regulado y determinado por la psiquiatría para patologizar la heterogeneidad humana (Vásquez-Rocca, 2011).

Así, cargada de elementos teóricos y políticos, la "antipsiquiatría clásica” de las décadas de 1960 y 1970 estableció una denuncia hacia el poder y función de la psiquiatría en la sociedad en un movimiento teórico-político con claras reivindicaciones y justificaciones sociales y políticas (Cea-Madrid y CastilloParada, 2016) y en un momento sociohistórico cargado de diversas reivindicaciones sociales, ideológicas, sindicales, culturales y políticas. 
Por tanto, si la patología tiene su origen en el contexto familiar y comunitario donde vive el sujeto, es allí donde debe producirse la terapia, y no en un contexto hospitalario (Pastor y Ovejero, 2009). Estos aires de reforma fueron importantes porque generan un nuevo modelo en la atención a las personas con enfermedad mental que evoluciona progresivamente del tratamiento clínico de la enfermedad a la atención integral en la propia comunidad, enfatizando su consideración como ciudadanos con derechos y deberes (López y Laviana-Cuetos, 2007).

Esta evolución se hace efectiva en España a mediados de los años ochenta con el proceso de reforma psiquiátrica, iniciada en 1985 con el Informe de la Comisión Ministerial para la Reforma Psiquiátrica, coincidiendo en un momento histórico de profundas transformaciones en todos los ámbitos y estamentos de la sociedad española (Desviat, 2000).

A partir de ese instante, se inicia el proceso de desinstitucionalización psiquiátrica y se asume el modelo de atención comunitaria. Su objetivo es articular la atención a los problemas psiquiátricos y sociales de estas personas en su propio entorno sociocomunitario, potenciando el mantenimiento e integración en el contexto familiar y social de la forma más normalizada posible (Ministerio de Sanidad y Consumo [MSC], 2007).

De esta manera, el marco idóneo para atender los trastornos mentales no es una institución cerrada sino la comunidad, de modo que se busca potenciar la autonomía de la persona como objetivo central de la pedagogía (Rosendal, 2013). Actualmente, los nuevos enfoques internacionales de atención enfatizan el concepto de recuperación (recovery) (Anthony, 2000; Scheyett et al., 2013), que hace referencia no sólo a la recuperación del trastorno, sino sobre todo a la recuperación del proyecto vital una vez que ha aparecido la enfermedad y la discapacidad (Garrido et al., 2008).

Desde este punto de vista, la educación adquiere vital importancia, ya que se reconoce como un derecho fundamental que debe posibilitar tanto la participación ciudadana en la vida económica, política y cultural, como el tratamiento educativo de los efectos, en forma de vulnerabilidad, desigualdad, exclusión, marginación e inadaptación social que la sociedad actual produce (García Molina, 2003).

Por otro lado, durante este periodo y hasta hoy en día, se han desarrollado diversos documentos y directrices que pretenden impulsar de manera definitiva la implantación de este modelo de inclusión social para las personas con TMS, tanto a nivel internacional como nacional.

Entre ellas podemos citar la Declaración de Helsinkiy el Plan de Acciones de 2005; el Libro Verde de la Salud Mental en Europa. Mejorar la salud mental 
de la población- Hacia una estrategia en salud mental en la Unión Europea, también de 2005; el "Pacto Europeo por la Salud Mental y el Bienestar" de 2008, importante en la lucha contra el estigma y la exclusión social; o el Plan de Acción sobre Salud Mental (2013-2020) de la Organización Mundial de la Salud (OMS).

Sin embargo, del análisis de la realidad se puede constatar no sólo el olvido y la ausencia de planteamientos pedagógicos en la definición de la política social en torno a la salud mental y al TMS, sino también que gran parte de estos documentos se han quedado en simples directrices sin llevarse a cabo. Además, la implantación real del modelo comunitario ha tenido sus luces y sombras (Espino, 2002), centrando los esfuerzos en la reorganización y estructuración del sistema sanitario, pero con una deficitaria red de apoyo (pocos alojamientos adaptados a las diferentes necesidades, falta de programas de inserción laboral, falta de programas comunitarios para la integración social).

Sumado a ello, la Administración Pública ha diluido gran parte de su responsabilidad en las entidades asociativas que ahora están pasando por graves problemas de liquidez, debido a los retrasos en los pagos por parte de las administraciones, a la incertidumbre sobre la continuidad de convenios y al desmantelamiento de la Ley de Promoción de la Autonomía y de Atención a las Personas en Situación de Dependencia, etc. (Pérez y Navarro, 2013).

En este sentido, debemos dejar patente la conexión de las políticas de carácter público con el contexto socioeconómico vigente en cada época. De ahí ese impulso inicial de principios de los años ochenta, caracterizado por un momento de profundas reformas en todos los ámbitos de la sociedad española; y de ahí también la escasa cobertura que ha tenido posteriormente el modelo comunitario. De este modo, las políticas económicas actuales de corte liberal cuestionan los criterios que hicieron posible la Europa del Bienestar, priorizan reducir el gasto social y contener el crecimiento del gasto público mediante políticas de restricción presupuestaria y privatización de servicios dependientes del Estado (Espino, 2002; Desviat, 2011).

Así, este contexto económico y político restrictivo para la sanidad pública afecta de forma especial a la salud mental, furgón de cola de los colectivos con necesidades sociosanitarias (Espino, 2002), y auténtica Cenicienta de los sistemas de salud y también de los servicios sociales, por lo que la familia y personas con trastorno mental emergen con grandes posibilidades de exclusión social, de modo que tendremos que darle la razón a Foucault (1976), cuando nos hace pensar que a través de todas las reformas psiquiátricas de los dos últimos siglos ha persistido el criterio de exclusión, cambiando de forma y de lugar. 
Esta nueva filosofía de atención nos debe situar ante unos retos y desafíos a los que es necesario dar respuestas orientadas a mejorar las realidades sociales desde una perspectiva holística, no restringida únicamente al ámbito clínico-psiquiátrico (Dimenstein et al., 2012). En dicho contexto, la Pedagogía Social debe mantener activa su capacidad para promover procesos de aprendizaje, de formación y desarrollo, con vocación de cambio y transformación social que contribuyan decisivamente al bienestar de las personas y a mejorar su calidad de vida (Caballo y Gradaílle, 2008).

De este modo, se explicita el deseo de transformar las condiciones sociales de existencia desde la sociedad, con la sociedad y para la sociedad (Caride, 2002 y 2005), que deben plantear modificaciones sustanciales en relación con el lugar que ocupan las personas en los procesos de acción social, siendo sujetos de la acción y no sólo objetos de aplicación de políticas más o menos paternalistas, de forma que la educación social, en palabras de Ortega-Esteban (1999: 18), "debe ante todo ayudar a ser y a convivir con los demás. Aprender a ser con los demás y a vivir juntos en comunidad”.

También se reafirma la importancia de la educación social en las políticas sociales, que oriente el quehacer pedagógico hacia la dinamización de recursos comunitarios, como la vivienda, la compensación de desigualdades, afrontar los problemas de inclusión social que las personas con TMS sufren, así como la búsqueda de nuevos cauces para su integración e inserción social y el desarrollo de la convivencia democrática (Caride, 2005).

Así pues, la atención a las personas con TMS no solamente debe englobar la visión de la Pedagogía/Educación Social de paliar y mejorar las situaciones surgidas de la marginación y la exclusión social, como se continúa realizando actualmente, sino que también sugiere la existencia de una intervención basada en la reciprocidad que ha de establecerse entre la dimensión social de la educación y la misión educativa de la sociedad (Caride et al., 2015).

En este sentido, "será fundamental que la Educación Social articule sus propuestas en torno a dos procesos, que deben ser considerados como indisociables y como punto de partida y de llegada: la construcción comunitaria y la participación democrática" (Caride, 2002: 107); ambos aspectos presentes en la nueva filosofía de atención a las personas con TMS, pero que sólo a través del ámbito socioeducativo puede llegar a implementarse de manera plena. Por ello, planteamos a la comunidad como espacio de intervención social, donde los ciudadanos amplían su protagonismo en la reflexión de su acción colectiva, van construyendo su propio discurso sobre lo necesario por transformar, buscan formas y procesos sociales que les sirven de modelos para sus acciones y respuestas a sus necesidades. 
Así, la acción comunitaria adquiere sentido cuando se desarrolla a partir de un colectivo humano que comparte un espacio y una conciencia de pertenencia, que genera procesos de vinculación y apoyo mutuo, y que activa voluntades de protagonismo en la mejora de su propia realidad (Gomá, 2008). Dichas acciones son fundamentales en el marco de trabajo socioeducativo para una verdadera recuperación del trastorno mental severo.

Por tanto, confluyen en este momento dos líneas que hasta ahora aparecían instauradas en el imaginario colectivo y profesional como paralelas y diferenciadas, que nunca llegaban a conectar, como son la atención al TMS y la Pedagogía Social.

Trastorno mental severo: concepto, necesidades y principales ámbitos de intervención pedagógica

Dentro de todo este nuevo paradigma de atención comunitaria se sitúan las personas con TMS. Su concepto, asumido por la mayor parte de las políticas y documentos mundiales en salud mental, se basa en la conjunción de tres dimensiones que hacen operativa su definición (MSC, 2007; Liberman, 1993; Ruggeri et al., 2000):

a) Diagnóstico, que suele incluir, fundamentalmente, esquizofrenia y otras psicosis delirantes (grupo diagnóstico mayoritario), psicosis afectivas y algunos tipos de trastornos de personalidad.

b) Duración y tratamiento, generalmente superior a dos años.

c) Funcionamiento global y presencia de discapacidad, significando alteraciones y déficits en varios aspectos funcionales como en la conducta social, las relaciones interpersonales, autocuidados, autonomía, ocio y tiempo libre, alojamiento y empleo.

Estas dimensiones se encuentran presentes en prácticamente la totalidad de la bibliografía revisada. Sin embargo, López y Laviana (2007) manifiestan la necesidad de añadir otra de carácter contextual, el estigma, una de las causas más importantes de limitación y restricción social y definido como "una marca de vergüenza, deshonra, desaprobación por la que el individuo es rechazado, discriminado y excluido de la participación en diversos ámbitos de la sociedad" (OMS, 2001: 16).

Las personas con TMS sufren altos niveles de estigma y discriminación (Whitley y Campbell, 2014). Dicho estigma termina por ser tan invalidante o más que los propios síntomas de la enfermedad, de modo que las actitudes de rechazo hacia estas personas y la consecuencia social negativa pueden levantar barreras adicionales que aumenten su riesgo de aislamiento y marginación. 
Por eso el apoyo sociocomunitario es fundamental, demostrando que bajos niveles de apoyo social se vinculan a niveles más altos de estigma y a niveles más bajos de calidad de vida (Chronister et al. 2013). Por tanto, como expone Garcés Trullenque (2010), sus problemas y necesidades desbordan el ámbito sanitario-psiquiátrico y se expresan en dimensiones de índole social: dificultades o restricciones para la actividad básica; dificultades o restricciones para la participación social; factores ambientales y factores personales que se traducen en una pobre calidad de vida (véase Tabla 1). ${ }^{1}$

A raíz de este tipo de problemáticas, se va generando toda una serie de necesidades que configuran las áreas principales de acción socioeducativa, siempre en el ámbito de la comunidad, como el apoyo a la integración, alojamiento y atención residencial; integración laboral, actividades de ocio y tiempo libre, tal y como se detalla en la Tabla 2.

En definitiva, el objetivo que tenemos por delante es que puedan recuperar su proyecto vital, para lo cual la comunidad adquiere un valor fundamental en el marco de intervención. Esto supone un cambio hacia la participación de diferentes agentes, principalmente socioeducativos y cuyo referente clave es la emancipación y transformación social, y es ahí donde la educación está estrechamente vinculada al desarrollo comunitario (García-Pérez, 2013a).

Se trata de conseguir un entorno comunitario favorable a la aceptación de las discapacidades que la enfermedad comporta y propiciar una optimización del uso de recursos disponibles en la comunidad, y en esto los profesionales socioeducativos tenemos una función primordial (García-Pérez y Torío-López, 2014a).

Así, pues, esa dimensión de funcionamiento global del TMS que destaca la OMS (2001), es la que, en buena medida, determina la diferenciación en las formas de intervención y distinción entre enfermo y un ciudadano más que necesita una serie de apoyos sociocomunitarios. Sobre esta cuestión, la comunidad internacional y el Estado español promueven diversas iniciativas orientadas a impulsar políticas de equiparación.

Especialmente importante es la "Convención sobre los Derechos de las personas con discapacidad” de la ONU de 2006, cuya pretensión es lograr el pleno desarrollo de las personas con discapacidad, mediante el ejercicio de sus derechos sociales, culturales, civiles y políticos. En España, siguiendo los postulados de la Convención, se elaboró la "Estrategia española sobre discapacidad 2012-2020", como fórmula para colaborar en la plena autonomía e inclusión del colectivo. Pero, de nuevo, la realidad nos asoma a una comunidad

1 Todas las tablas se encuentran en el Anexo, al final del presente artículo (Nota de los editores). 
en la que el TMS no se asocia con diversidad funcional, sino con una enfermedad que debe ser tratada bajo los parámetros médicos, y no desde una provisión de apoyos desde los Servicios Sociales.

Por ello, estas actuaciones de inclusión social en la comunidad no son posibles si realmente las personas con TMS no viven en la comunidad de manera efectiva. Por tanto, facilitar el alojamiento es el primer elemento básico de intervención sobre el cual actuar, ofreciendo, en primer lugar, una estabilidad residencial acorde con la elección y preferencias de los usuarios y, a partir de ahí, establecer los apoyos vitales, educativos, laborales, sociales y de ocio adecuados.

\section{Alojamiento con apoyo como base esencial de recuperación}

La vivienda es una necesidad básica humana y universal, es el entorno donde se comprometen más rutinas cotidianas de cuidado personal y de relaciones sociales, familiares e íntimas, por lo que la sensación de hogar es crucial para la salud mental positiva de todo ciudadano. Las personas con TMS se enfrentan a los mismos problemas de vivienda que otros grupos de la comunidad. Sin embargo, su situación puede ser muy insegura o precaria, con graves dificultades para acceder y mantenerse en una vivienda digna y adecuada a sus demandas y deseos.

Según apunta Ridgway (2008), las personas con discapacidades psiquiátricas tienen un riesgo diez veces superior al de la población general de convertirse en personas sin hogar. En esta circunstancia influyen diferentes factores (López Álvarez et al., 2004; Nelson, Aubry y Hutchinson, 2013; Ridgway, 2008): dificultad en el proceso de búsqueda, acceso y mantenimiento de vivienda; discriminación por motivos del estigma que acompaña a la enfermedad mental; insuficientes ingresos económicos; insuficiente planificación social en el marco de la política de desinstitucionalización, que se traduce en una inadecuada o limitada organización de servicios residenciales comunitarios.

Además, el no disponer de una vivienda adecuada genera una serie de consecuencias negativas de cara a la atención e integración sociocomunitaria de las personas con TMS y afecta a todo el modelo de atención tanto sanitario como social: incremento de reingresos hospitalarios; sobrecarga familiar; dificultades de integración comunitaria; incremento de personas con TMS en situación de marginación sin hogar (Asociación Española de Neuropsiquiatría [AEN], 2002; Rodríguez, 2002). 
En este sentido, la adquisición de una vivienda digna proporciona un punto de inflexión que permite a la gente comenzar a trabajar en su recuperación y, a partir de ahí, lograr otros objetivos de su vida (Ridgway, 2008). Asimismo, como indican Rogers et al. (2009), aparte del tratamiento, probablemente no hay área de servicios más importante para la recuperación de las personas con TMS que la prestación de servicios de vivienda.

Así, la creación y desarrollo de estos dispositivos de alojamiento ha experimentado una evolución que ha ido pasando desde las "casas a medio camino" o "viviendas de transición" (Halfway House), con un importante contenido terapéutico y rehabilitador, hasta la filosofía denominada supported housing o alojamiento con apoyo, sobre la que se basa el modelo de atención residencial considerado actualmente la referencia mundial, gracias a la evidencia de sus resultados. Se trata del conocido housing first y del programa "Pathways to Housing Program" desarrollado por Tsemberis y Eisenberg (2000) en Nueva York y que se está exportando por todo Estados Unidos y varios países europeos como Dinamarca, Finlandia, Francia y Suecia (Pleace y Wallace, 2011).

\section{Evolución de la atención residencial para personas con trastorno mental severo}

Los últimos 30 años han sido testigos de un florecimiento de los servicios de vivienda y los modelos de prestación de servicios, impulsados por la desinstitucionalización y el movimiento hacia la integración comunitaria de las personas con TMS. De manera habitual, los programas residenciales han experimentado una evolución similar, que podemos presentar en tres fases sucesivas (Fakhoury et al. 2002; López et al. 2004; Nelson, 2010; Nelson et al. 2013; Newman, 2001; Ridgway, 2008; Rogers et al. 2009):

1. En un primer momento, se crean las "casas a medio camino" o "viviendas de transición" (Halfway house), que pretenden servir de paso para la vida en la comunidad, prolongando la atención sanitaria con un importante contenido terapéutico y rehabilitador, dentro de un modelo de atención custodial. Respecto a este modelo, se evidenció que reducía el funcionamiento social independiente, aumentando la actividad asistida (Segal y Kotler, 1993) y mostrando menos beneficios personales, de participación e independencia que otros alojamientos con apoyo más comunitario (Nelson et al., 1998).

2. En un segundo momento, los programas empiezan a adquirir independencia de las redes de servicios sanitarios, a la vez que adoptan una estructura que combina distintas alternativas graduadas, según niveles de supervisión y apoyo, pasando los usuarios, en ocasiones, por más de diez 
tipos de dispositivos diferentes (Carling, 1993; López et al., 2004; Ogilvie, 1997). Es el modelo conocido como "continuum residencial", "continuum lineal" o "modelo escalera". Se trataba de ir articulando una especie de escalera de recursos intermedios entre el hospital y la comunidad que fueran ofreciendo servicios basados en un funcionamiento prototípico según el perfil funcional de los enfermos (Ridgway, 2008), hasta la vida independiente. Las críticas a esta filosofía de atención residencial resaltan el poco margen que habitualmente deja a la iniciativa y preferencias de los usuarios, y las dificultades generadas a la hora de hacer efectiva la movilidad que fundamenta el modelo (Geller y Fisher, 1993; Nelson et al., 2013; Ridgway, 2008; Ridway y Zipple, 1990). Sin embargo, sigue siendo el modelo más característico en la atención residencial a personas con TMS.

3. Estas críticas del modelo lineal determinan la aparición, básicamente en Estados Unidos y hace algo más de una década, de una tercera perspectiva y de un modelo alternativo, denominado supported housing, apoyo residencial o alojamiento con apoyo, con un sistema de apoyo flexible y continuado, donde las personas con TMS ejercen el control de su hogar, que responde a su elección y preferencias. Se trata de un programa que lo primero que ofrece es una vivienda subvencionada, estable y permanente antes que otras intervenciones, y luego lo combina con otros servicios y apoyos individualizados en función de las necesidades de la persona (Tsemberis y Eisenberg, 2000).

Sin embargo, aun destacando sus buenos resultados, algunos autores consideran que es un modelo reducido a la atención a los pobres y a personas sin hogar (Desviat, 2011), cuya principal motivación política para su implantación es la reducción de costos (Stanhope y Dunn, 2011). En este caso, las ideas o principios básicos que sustentan el modelo incluyen una serie de premisas elementales como la necesidad básica de hogar, que puedan elegir su vivienda acorde con sus preferencias, que tengan el control de sus actividades y estilo de vida, etc. (López et al., 2004; Nelson et al., 2013; Ridgway, 2008; Ridgway y Zipple, 1990) (véase Tabla 3).

En este sentido, pocos estudios han comparado directamente ambos modelos; incluso los existentes no aclaran las evidencias y resultados diferenciales (Goldfinger et al., 1999). Otros estudios, como los de Tsemberis y Eisenberg (2000) o Siegel et al. (2006), muestran una mayor estabilidad en la vivienda, una mejor autonomía y mayores niveles de satisfacción, mayor participación comunitaria y reducción de síntomas en los usuarios del supported housing, debido a que hacen efectivas sus preferencias (Nelson, 2010; Rogers et al., 2009). Por tanto, mejoran el bienestar cuanto menor sea el nivel de restricción de la vivienda y también provoca un menor coste económico al sistema (Pleace y Wallace, 2011). 
Resultados de los programas de alojamiento con apoyo para personas con TMS

La literatura científica del alojamiento con apoyo para personas con TMS constatan los límites de la evidencia y la falta general de pruebas sólidas en todo el sector (Chilvers et al., 2010; Fakhoury et al., 2002; O'Malley y Croucher 2005). ${ }^{2}$ Pese a ello, hay información suficiente para afirmar que los distintos programas residenciales se asocian con resultados positivos para las personas con TMS (García-Pérez y Torío-López, 2014a; Hubley et al., 2014; López et al., 2004; Nelson, 2010; Nelson et al., 2013; Newman, 2001; Pleace y Wallace, 2011; Pleace y Quilgars, 2013; Ogilvie, 1997; Rogers et al., 2009; Ridgway, 2008; Tsemberis, 2010). Destacamos, entre otros, los siguientes:

- Son capaces de mantener en la comunidad a un número considerable de personas con trastorno mental severo.

- Ofrecen cierta estabilidad residencial.

- Mejoran el funcionamiento y la integración social.

- Reducen la incidencia de hospitalización y los síntomas psiquiátricos.

- Aumentan el nivel de funcionamiento básico y social (incremento de actividad social y actividades vida diaria).

- Mejoran el nivel de vinculación con los recursos comunitarios.

- Aumentan la satisfacción con su propia vida.

- Incrementan la calidad de vida y producen un gran sentido y pertenencia de hogar.

- Son rentables: reducen costes debido a un menor uso de servicios, pues mejoran de su enfermedad, están más satisfechos de su situación vital, lo cual ocasiona un menor número de ingresos hospitalarios y, en caso de tenerlos, los días de ingreso son menores, etcétera.

2 Eso hace que tanto clínicos como gestores públicos duden o no tengan claro el potencial de las viviendas o programas residenciales para TMS y cómo éstas pueden influir de forma positiva en resultados importantes para la salud, máxime en un periodo en el que las decisiones políticas y administrativas en cuanto a la financiación y puesta en marcha de programas depende y se basa en la tan buscada evidencia científica, fundamentada en el positivismo y en la generalización de resultados por medio de resultados estadísticos (Stanhope y Dunn, 2011). En este sentido, revisiones como la de Chilvers et al. (2010) no encuentra ninguna evidencia, debido a que basa su estudio únicamente en investigaciones con metodología experimental con ensayos aleatorios. Sin embargo, otras como Rogers et al. (2009) o Nelson (2010), plantean que existe literatura importante y significativa que podría ser útil para los interesados, tanto para usuarios como trabajadores y responsables de los programas, así como para otros componentes del campo de la salud mental y la intervención socioeducativa. 
Naturalmente, estos resultados dependen de diferentes variables asociadas al alojamiento: ubicación de la vivienda, funciones y servicios prestados; carácter temporal o definitivo del alojamiento; número y características del personal de apoyo; ambiente abierto o más restrictivo; recursos del entorno; resistencias vecinales, etc. De este modo se ha demostrado que el hecho de residir en una vivienda de baja calidad o inadecuada incrementa los riesgos de deterioro de la actividad, reduce la calidad de vida y aumenta el número de rehospitalizaciones (Fakhoury et al., 2002).

Por tanto, la vivienda y los apoyos ofrecidos en ella son un elemento crucial en la recuperación de las personas con TMS y en su inserción social, teniendo consecuencias positivas o negativas en función de la elección o preferencia de sus usuarios y del tipo de programa que se implemente en ellas.

\section{A modo de conclusión}

El cambio de consideración de la enfermedad mental y, por ende, de abordar la atención a sus necesidades y problemáticas, hace patente la necesidad de realizar una intervención pedagógica, dentro del marco de atención comunitaria. Sin embargo, del análisis de la realidad actual, se puede constatar el olvido y la ausencia de planteamientos socioeducativos en la definición de la política social en torno a la salud mental y al TMS.

Además, la implantación real del modelo comunitario ha quedado a medio camino, dejando de lado la parte más social y educativa, de apoyo comunitario, laboral y residencial y continuando con un modelo clínico en el que gran parte de los programas "sociales" destinados a estas personas y/o a sus familias provienen de los servicios de salud mental. Así pues, debe huirse de las soluciones y tratamientos parciales, e ir hacia acciones globales y holísticas, pues como manifiesta Desviat (2011: 292):

lo comunitario no es la rotación por los centros de salud mental [...], ni un programa de atención a los pobres, como ha quedado reducido en Estados Unidos [...], lo comunitario es el trabajo en red, es la acción en un territorio en continua interacción con sus ciudadanos y sus organizaciones. Una ciudadanía que forma parte del proceso, que hace suyo el proceso asistencial.

Si la integración de las personas con trastorno mental severo en la comunidad es un principio fundamental, valor y meta de la política de salud mental contemporánea (Wong et al., 2011), la vivienda emerge como el eje principal sobre el cual fundamentar esa integración y manejar su recuperación.

Por una parte, ha quedado demostrado que son recursos rehabilitadores efectivos, mejorando aspectos clínicos y su funcionamiento básico, pero, además, 
incrementan la calidad de vida y su sentimiento de pertenencia a la comunidad, así como sus relaciones sociales. Por otro lado, resulta de gran importancia el hecho de que los propios usuarios perciben mejoría, incrementando su autoestima y su autoconfianza para hacer cualquier actividad, con la seguridad de tener una vivienda y los apoyos necesarios para su realización.

Así, la finalidad última de los servicios residenciales para personas con TMS es servir como punto de partida para conseguir el cambio de rol de "cliente" a un papel de ciudadano por medio de la vivienda (Newman y Goldman, 2008), así como mediante la educación y las oportunidades de trabajo (Piat y Sabetti, 2010).

Sin embargo, no solamente se convierte en un recurso en el que puede haber intervención directa ser el enlace con el resto de servicios comunitarios, sino que sitúa directamente a la persona en la comunidad, en un barrio sobre el cual construir las bases de una participación ciudadana por parte de todos, de modo que se trabaje de forma conjunta en una simbiosis de fomento de la ciudadanía, mejorando no sólo su situación personal, sino la reducción del estigma, la eliminación de etiquetas negativas por parte de la sociedad. Esto revierte en la reducción del rechazo social y afecta directamente a la mejora de su inclusión social, y así sucesivamente, en un círculo de mejoras mutuas que genera esa construcción comunitaria.

Para la construcción de este "círculo comunitario de recuperación" es necesario, en primer lugar, la implantación de una política pública que realmente apoye un modelo estable de apoyo a la vivienda y de unos sistemas socioeducativos, sanitarios y laborales coordinados que garanticen prestaciones universales y equitativas, donde funcione la complementariedad y la colaboración y no la competencia y la insolidaridad (Desviat, 2011).

Este reclamo parece convertirse en una utopía en un momento de recortes sociales y desmantelamiento del Estado de Bienestar en el contexto europeo, cuyos dos principios fundacionales, solidaridad y ciudadanía social (RománBrugnoli et al., 2014), se están resquebrajando. En este sentido, los efectos sociales de la crisis en España son bastante evidentes tanto desde el punto de vista individual como colectivo, incrementando el riesgo de fractura social.

La situación de crisis económica conduce a un incremento de necesidades sociales relacionadas con el empleo, la vivienda, la renta y la alimentación y afecta especialmente a quienes se encuentran en peor situación de pobreza y exclusión social, como las personas con TMS (García-Pérez, 2013b). Ante este panorama, muchas personas con TMS viven en la pobreza crónica y no tienen los recursos adecuados para conseguir una vivienda digna. 
Ello se traduce, a menudo, en que los individuos se ven obligados a utilizar el sistema de refugios o a vivir en las calles (Nelson et al., 2013; Ridgway, 2008), por lo que debemos enfatizar su atención desde una forma multidimensional: social, económica y ambiental (Martínez-Treviño et al., 2014). Si esta situación delicada de las personas con TMS no es suficiente para implantar estos sistemas de apoyo, quizás, en un contexto neoliberal como el actual, el hecho de que, por ejemplo, las viviendas con apoyo reducen costes a los servicios públicos sea razón suficiente para llevarlo a cabo, tal y como ocurrió con el programa Housing First en Estados Unidos (Stanhope y Dunn, 2011).

Por otro lado, debemos trascender el concepto de ciudadanía que se promueve desde las políticas públicas de atención al TMS, que en la práctica está basado, casi simplemente, en la concepción de estatus legal, y no como posibilidad real de ejercer ciertos deberes y derechos, siguiendo el cuestionamiento público a la capacidad de los Estado-nación modernos para ofrecer oportunidades efectivas e igualitarias para sus habitantes (Gómez-Urrutia, 2014; Nussbaum, 2011). De poco sirve la proliferación de legislaciones, recomendaciones y estrategias -señaladas en líneas anteriores-, si no vienen acompañadas de recursos y personal, además de una modificación de la estructura social y cultural, garantizando unos niveles mínimos para todos los ciudadanos.

En definitiva, se hace pertinente no solamente un cambio paliativo, que es lo que ha sucedido, de mejora de un sistema existente, eminentemente sanitario y rehabilitador, sino un cambio transformador. Siguiendo la propuesta de Nelson (2010), las personas con problemas de salud mental no sólo deben estar en la comunidad, sino ser miembros valiosos de la comunidad.

Esta nueva posición exige un concepto de ciudadanía social que promueva las condiciones de igualdad de oportunidades y equidad en el acceso y en el tratamiento en el espacio público y en sus instituciones; una ciudadanía inclusiva que promueva la justicia social facilitando la incorporación de las personas con TMS, para que sus intereses sean representados, sus derechos respetados y sus necesidades individuales y colectivas atendidas.

Aun conociendo la imposibilidad de dar una atención ilimitada de recursos, las políticas públicas deben garantizar una amplia gama de servicios alternativos de vivienda, una vez demostrados sus efectos positivos, como un derecho básico de la vida, más allá de un medio terapéutico, además de evidenciarse su menor coste respecto al sistema actual de atención médica.

Así pues, el desarrollo de este escrito muestra la confluencia de los fundamentos necesarios que justifican la importancia de una intervención socioeducativa en diversas áreas de actuación, recreando escenarios de acción pedagógica en un sector de la población fuertemente estigmatizado. 
Por tanto, la vivienda ejerce como elemento aglutinador sobre el cual giren las acciones a efectuar de una forma coordinada, comunitaria e integral, con un gran abanico de posibilidades centradas en diversos apoyos, en función de las necesidades y demandas de las personas con TMS y sus preferencias:

- Desarrollo comunitario y educación cívico-social: con intervenciones en el área de autonomía en el entorno comunitario y desenvolvimiento en el medio, programas en el área de convivencia, familiares, de pareja, etc. Dentro de este epígrafe podemos incluir cualquier elemento que favorezca la participación social y el trabajo dirigido a la erradicación del estigma y degradación social que sufren las personas con $T M S$. En este sentido, toda interacción comunitaria puede generar respuestas positivas o negativas, de modo que muchas interacciones pueden provocar síntomas de estrés, ansiedad, y ser perjudiciales para el desarrollo del trastorno mental severo, por lo que se va generando una respuesta de evitación. Llegados a este punto, los usuarios llegan a asimilar lo que Corrigan et al. (2009) denominaron el modelo de: “¿Por qué intentarlo?”. El modelo sugiere que, como resultado del estigma internalizado, personas con TMS reducen la autoestima y la autoeficacia, que podrían conducir a evitar el logro de sus objetivos vitales. Por lo tanto, las personas con TMS que son conscientes del estigma público hacia la enfermedad mental y adoptan esas actitudes estigmatizantes, pueden cuestionar su capacidad para participar en la comunidad y conseguir la inclusión social (García-Pérez y ToríoLópez, 2014b). Apoyo educativo: en referencia a programas con un componente escolar de apoyo en educación formal, como el conocido supported education o apoyo educativo de Mowbray et al. (2005), con grandes resultados en la consecución de estudios superiores de personas con TMS y que revierten directamente en la mejora personal y social de sus usuarios.

- Apoyo formativo-ocupacional y de inserción laboral: con acciones dirigidas al desarrollo de su orientación vocacional, los hábitos básicos de trabajo, las habilidades de ajuste al entorno laboral, apoyo formativo en todo tipo de propuestas de educación no formal, que enlazan con el concepto de aprendizaje para toda la vida, entrenamiento en la búsqueda de empleo, el acceso y mantenimiento en el puesto de trabajo, la inserción en el mercado laboral ordinario, empleo con apoyo, empleo protegido, etc. Todos estos aspectos llevan consigo un trabajo formativo con el mundo empresarial, con la comunidad y el contexto donde se encuadran las acciones, y que se revierten en la mejora de 
habilidades comunicativas, sociales, aumento de redes sociales, con intervenciones en el área de autonomía en el entorno comunitario y desenvolvimiento en el medio, a la par que realizan un uso adecuado de su tiempo libre.

- Ocio y animación sociocultural: En este caso debemos procurar que estas personas puedan tener un "ocio normalizado", lo cual también necesita de un cierto aprendizaje para su elección y disfrute, dotándoles de recursos personales a través de diferentes actividades socioeducativas en una plena integración con los recursos comunitarios. El ocio se convierte en un cauce de participación, un elemento socializador y de aprendizaje de valores culturales y cohesión, así como fuente e impulso de transformación comunitaria. Además, se ha demostrado su influencia en la recuperación de las personas con TMS (Iwasaki et al., 2014).

Para la consecución de estos retos, es imprescindible trabajar en y con la comunidad, en y con el barrio, el entorno, la ciudad. La ciudad y el barrio donde viven se convierten en un agente rehabilitador y en un agente educativo en el que el ser humano tiene que abordar constantemente situaciones nuevas y difíciles de prever. En este contexto, la educación social promueve estrategias didácticas que fomentan la autonomía personal, de modo que las relaciones que se producen en la vida cotidiana de las personas son, al mismo tiempo, vehículos, contextos y contenidos de las acciones sociopedagógicas (Úcar, 2013).

De este modo, la educación debe ser un instrumento de ayuda y respuesta a las necesidades cotidianas y concretas de la población, convirtiendo a la sociedad en un enorme potencial formativo, como lugar de intercambios culturales y como escuela de civismo, democracia, solidaridad y participación (García-Pérez y Torío-López, 2014b). Por lo tanto, debemos repensar la pedagogía social y la generación de nuevos enfoques más acordes con la complejidad de la realidad actual; el campo natural de intervención es la vida cotidiana de las personas, no una institución específica.

En este sentido, la Pedagogía Social puede ayudar también a las personas con TMS, asesorar, orientar y apoyar en los procesos de empoderamiento que les dotan de recursos para mejorar su calidad de vida. Por eso, los apoyos prestados a los usuarios del alojamiento con apoyo han de ser individualizados en función de las preferencias y necesidades expresadas por las personas con TMS, en una relación y toma de decisiones conjunta entre profesional socioeducativo y participante, implementando las metodologías participativas en salud mental (Cea-Madrid, 2015). 
Finalmente, la configuración de una política de acción y de bienestar social de estas características necesita de diversas perspectivas, como ha quedado patente, entre las cuales la dimensión pedagógica resulta básica y fundamental (March, 1988). Así pues, se abre un nuevo marco de acción, tanto desde el punto de vista teórico como práctico y se constata la relevancia de la Pedagogía Social y la Educación Social en la labor de recuperación de estas personas y en la mejora de su bienestar y calidad de vida, mediante acciones socioeducativas que tienen como eje central la vivienda.

\section{Referencias}

Asociación Española de Neuropsiquiatría (AEN) (2002), Rehabilitación psicosocial del trastorno mental severo. Situación actual y recomendaciones. Cuadernos Técnicos, núm. 6, España: Asociación Española de Neuropsiquiatría.

Anthony, William A. (2000), "Recovery-oriented service systems: setting some system level standards", en Psychiatric Rehabilitation Journal, vol. 24, núm. 2, Estados Unidos: American Psychological Association.

Caballo, Belén y Rita Gradaílle (2008), "La educación social como práctica mediadora en las relaciones escuela-comunidad local", en Pedagogía Social. Revista Interuniversitaria, núm. 15, España: Sociedad Iberoamericana de Pedagogía Social (SIPS).

Caride, José A. (2002), "La Pedagogía Social en España”, en Núñez, Violeta [coord.], La educación en tiempos de incertidumbre: las apuestas de la Pedagogía Social, España: Gedisa.

Caride, José A. (2005), Las fronteras de la Pedagogía Social, España: Gedisa.

Caride, José A. et al. (2015), "De la pedagogía social como educación, a la educación social como pedagogía", en Perfiles Educativos, vol. XXXVII, núm. 48, México: ISUEUniversidad Nacional Autónoma de México.

Carling, Paul J. (1993), "Housing and supports for persons with mental illness: emerging approaches to research and practice", en Hospital and Community Psychiatry, vol. 44, núm. 5, USA: American Psychological Association.

Cea-Madrid, Juan Carlos (2015), "Metodologías participativas en Salud Mental: alternativas y perspectivas de emancipación social más allá del modelo clínico y comunitario", en Teoría Crítica de la Psicología, núm. 5, México: Universidad Michoacana de San Nicolás de Hidalgo.

Cea-Madrid, Juan Carlos y Tatiana Castillo-Parada (2016), "Materiales para una historia de la antipsiquiatría: balance y perspectivas", en Teoria Crítica de la Psicología, núm. 8, México: Universidad Michoacana de San Nicolás de Hidalgo.

Chilvers, Rupatharshini et al. (2010), "Supported housing for people with severe mental disorders (Review)”, en Cochrane Schizophrenia Group, núm. 12, Chichester, Reino Unido: Wiley.

Chronister, Julie et al. (2013), "The role of stigma coping and social support in mediating the effect of societal stigma on internalized stigma, mental health recovery, and quality of life among people with serious mental illness", en Journal of Community Psychology, vol. 41, núm. 5, USA: Wiley. 
Comisión de las Comunidades Europeas (2005), Libro Verde Mejorar la salud mental de la población. Hacia una estrategia de la Unión Europea en materia de salud mental. Disponible en:

http://ec.europa.eu/health/archive/ph_determinants/life_style/mental/green_paper/ mental_gp_es.pdf [14 de julio de 2015].

Corrigan, Patrick W. et al. (2009), "Self-stigma and the "why try" effect: impact on life goals and evidence-based practices", en World Psychiatry, vol. 8, núm. 2, Ginebra: Wiley.

Desviat, Manuel (2000), "La asistencia de las psicosis en España o hacia dónde va la reforma psiquiátrica”, en Rivas Guerrero, Fabio [coord.], La Psicosis en la Comunidad, Madrid: Asociación Española de Neuropsiquiatría.

Desviat, Manuel (2006), "La antipsiquiatría: crítica a la razón psiquiátrica”, en Norte de Salud Mental, núm. 25, País Vasco: Osasun Mentalaren Elkartea, Asociación de Salud Mental.

Desviat, Manuel (2011), "Panorama actual de las políticas de bienestar y la reforma psiquiátrica en España”, en Estudos de Psicologia, vol. 16, núm. 3, Brasil: Scielo.

Dimenstein, Magda et al. (2012), "Participación y redes de cuidado entre usuarios de servicios de salud mental en el nordeste brasileño: mapeando dispositivos de reinserción social”, en Psicología desde el Caribe, vol. 29, núm. 3, Colombia: Universidad del Norte.

Espino, Antonio (2002), "Análisis del estado actual de la reforma psiquiátrica: debilidades y fortalezas. amenazas y oportunidades”, en Revista AEN, vol. XXII, núm. 81, España: Asociación Española de Neuropsiquiatría.

Fakhoury, Walid K. H. et al. (2002), "Research in supporting housing”, en Social Psychiatry and Psychiatric Epidemiology, vol. 37, núm. 7, USA: Springer.

Foucault, Michel (1976), Historia de la locura en la Época Clásica, México: Fondo de Cultura Económica.

Garcés Trullenque, Eva María (2010), “El Trabajo Social en Salud Mental”, en Cuadernos de Trabajo Social, núm. 23, España: Universidad Complutense de Madrid.

García-Molina, José (2003), “Educación Social: ¿ profesión educativa o empleo social?”, en De nuevo, la Educación Social, Madrid: Dykinson.

García-Pérez, Omar (2013a), "Viviendas supervisadas para personas con trastorno mental severo en Asturias: ‘ambiente restrictivo o abiertas a la comunidad?”, en Pedagogía Social. Revista Interuniversitaria, núm. 22, España: Sociedad Iberoamericana de Pedagogía Social.

García-Pérez, Omar (2013b), "El rol del ciudadano de la persona con trastorno mental severo: preferencias y poder de elección sobre la vivienda”, en Torío-López, Susana et al. [eds.], La Crisis Social y el Estado de Bienestar: las respuestas de la Pedagogía Social, España: Servicio de Publicaciones de la Universidad de Oviedo.

García-Pérez, Omar y Susana Torío-López (2014a), "Funcionamiento básico y social de los usuarios de las viviendas supervisadas para personas con trastorno mental severo en Asturias: necesidad de una intervención pedagógica”, en Revista Complutense de Educación, vol. 25, núm. 2, España: Universidad Complutense de Madrid.

García-Pérez, Omar y Susana Torío López (2014b), "Alojamiento con apoyo para personas con trastorno mental severo: ¿Participan realmente en la comunidad?”, en Del PozoSerrano, Francisco José y Carlos Peláez-Paz [coords.], Educación Social en situaciones de riesgo y conflicto en Iberoamérica, España: Universidad Complutense de Madrid. 
Garrido et al. (2008), "Buscando la reconstrucción personal, retomando el control de la propia vida. Un diseño para favorecer procesos de "recovery" y "empowerment", en Informaciones Psiquiátricas, núm. 194, España: Hospital Benito Menni.

Geller, Jeffrey L. y William H. Fisher (1993), "The linear continuum of transitional residence: debuking the myth", en American Journal of Psychiatry, vol. 150, núm. 7, USA: American Psychological Association.

Geneyro, Silvia Carolina y Francisco Javier Tirado (2015), “Diagnóstico clínico en biopsiquiatría: de la hermenéutica clínica a la traducción psicofarmacológica”, en Sociología y Tecnociencia, vol. 4, núm. 1-2, España: Universidad de Valladolid.

Goldfinger, Stephen et al. (1999), "Predicting homelessness after rehousing: A longitudinal study of mentally ill adults", en Psychiatric Services, vol. 50, núm. 5, USA: American Psychological Association.

Gomá, Ricard (2008), “La acción comunitaria: transformación social y construcción de ciudadanía”, en RES. Revista de Educación Social, núm. 7, España: Asociación Estatal de Educación Social.

Gómez-Urrutia, Verónica (2014), “Modelos de ciudadanía: discursos sobre roles femeninos en la legislación chilena”, en Convergencia Revista de Ciencias Sociales, núm. 66, México: Universidad Autónoma del Estado de México.

Hubley, Anita M. et al. (2014), "Subjective quality of life among individuals who are Homeless: a review of current knowledge”, en Social Indicators Research, núm. 115, Nueva York: Springer.

Iwasaki, Yoshitaka et al. (2014), "Role of leisure in recovery from mental illness", en American Journal of Psychiatric Rehabilitation, vol. 17, núm. 2, Philadelphia: Taylor \& Francis.

Liberman, Robert Paul (1993), Rehabilitación integral del enfermo mental crónico, España: Martínez Roca.

López-Álvarez, Marcelino et al. (2004), "Los programas residenciales para personas con trastorno mental severo. Revisión y propuestas", en Archivos de Psiquiatría, vol. 67, núm. 2, España: Tricastelia.

López-Álvarez, Marcelino y Margarita Laviana-Cuetos (2007), “Rehabilitación, apoyo social y atención comunitaria a personas con trastorno mental grave. Propuestas desde Andalucía”, en Revista AEN, vol. XXVII, núm. 99, España: Asociación Española de Neuropsiquiatría.

Macpherson, Rob et al. (2004), "Supported accommodation for people with severe mental illness: a review”, en Advances in Psychiatric Treatment, núm. 10, Reino Unido: Royal College of Psychiatrists.

March, Martí Xavier (1988), “La intervención pedagógico-social en el ámbito de la inadaptación social: hacia una pedagogía de la inadaptación social", en Pedagogía Social. Revista Interuniversitaria, núm. 3, España: SIPS.

Martínez-Treviño et al. (2014), "El referente de la pobreza en el discurso de la ONU sobre el desarrollo sostenible”, en Convergencia Revista de Ciencias Sociales, núm. 66, México: Universidad Autónoma del Estado de México.

Ministerio de Sanidad, Política Social e Igualdad (2012), Estrategia Española sobre Discapacidad 2012-2020, Madrid: Gobierno de España. Disponible en: http:// www.lamoncloa.gob.es/espana/eh 14/social/Documents/estrategia_espanola_ discapacidad_2012_2020.pdf [14 de julio de 2015]. 
Omar García-Pérez, José Vicente Peña-Calvo y Susana Torío-López. Atención socioeducativa y trastorno mental severo: la vivienda como base de intervención

MSC (2007), Estrategia en Salud Mental del Sistema Nacional de Salud, 2006, España: Ministerio de Sanidad y Consumo.

Morales-Ramírez, Francisco (2012), "La recepción de la antipsiquiatría en México entre las décadas de 1970 y 1980", en Temas de Historia de la Psiquiatría Argentina, vol. XV, núm. 31, Argentina: Polemos.

Mowbray, Carol T. et al. (2005), "Supported education for adults with psychiatric disabilities: an innovation for social work and psychosocial rehabilitation practice”, en Journal of Social Work, vol. 50, núm. 1, Reino Unido: Sage Publications.

Nelson, Geoffrey (2010), "Housing for people with serious mental illness: approaches, evidence, and transformative change", en Journal of Sociology \& Social Welfare, vol. XXXVII, núm. 4, USA: Western Michigan University.

Nelson, Geoffrey et al. (1998), "The relationship between housing characteristics, emotional well-being, and the personal empowerment of psychiatric consumer/survivors", en Community Mental Health Journal, vol. 34, núm. 1, Nueva York: Springer.

Nelson, Geoffrey et al. (2013), "Housing and Mental Health", en Stone, J. H. y M. Blouin [eds.], International Encyclopedia of Rehabilitation. Disponible en: http://cirrie. buffalo.edu/encyclopedia/en/article/132/ [13 de enero de 2013].

Newman, Sandra (2001), "Housing attributes and serious mental illness: implications for research and practice”, en Psychiatric Services, vol. 52, núm. 10, USA: American Psychological Association.

Newman, Sandra y Howard Goldman (2008), "Putting Housing First, Making Housing Last: Housing Policy for Persons with Severe Mental Illness", en American Journal of Psychiatry, vol. 165, núm. 10, USA: American Psychological Association.

Nussbaum, Martha (2011), Creating capabilities, Cambridge: Harvard University Press.

Ogilvie, Rita J. (1997), “The state of supported housing for mental health consumers: A literature review”, en Psychiatric Rehabilitation Journal, vol. 21, núm. 2, USA: American Psychological Association.

O'Malley, Lisa y Karen Croucher (2005), "Supported housing services for people with mental health problems”, en Housing Studies, vol. 20, núm. 5, Reino Unido: Taylor \& Francis.

OMS (2001), Clasificación Internacional del Funcionamiento, de la discapacidad y de la salud: CIF, España: Ministerio de Trabajo y Asuntos Sociales.

OMS (2005), Mental Health: Facing the Challenges, Building Solutions: report from the WHO European Ministerial Conference of Helsinki, Copenhagen: WHO Regional Office for Europe. Disponible en: http://www.msssi.gob.es/organizacion/sns/ planCalidadSNS/pdf/excelencia/salud_mental/opsc_est17.pdf.pdf [14 de julio de 2015].

OMS (2008), "Pacto Europeo para la Salud Mental y el Bienestar", en Conferencia de alto nivel de la UE "Juntos por la salud mental y el bienestar", Bruselas, 12-13 junio. Disponible en: ec.europa.eu/health/mental_health/docs/mhpact_es.pdf [14 julio de 2015].

OMS (2013), Plan de Acción sobre Salud Mental 2013-2020, Ginebra: OMS. Disponible en: http://www.who.int/iris/bitstream/10665/97488/1/9789243506029_spa.pdf. [14 de julio de 2015].

ONU (2006), Convención Internacional sobre los Derechos de las Personas con Discapacidad. Disponible en www.un.org/esa/socdev/enable/documents/tccconvs.pdf [ 14 de julio de 2015]. 
Ortega-Esteban, José (2005), "Pedagogía Social y Pedagogía Escolar: la Educación Social en la Escuela”, en Revista de Educación, núm. 336, España: Ministerio de Educación.

Ortega-Esteban, José (1999), "Educación social especializada. Concepto y profesión”, en Educación Social Especializada, Barcelona: Ariel.

Pastor, Juan y Anastasio Ovejero (2009), "Historia de la locura en la época clásica y el movimiento antipsiquiátrico”, en Revista de Historia de la Psicología, vol. 30, núm. 2-3, Valencia: Publicacions Universitat Valencia.

Pérez, Manuel y Luis Navarro (2013), "El Tercer Sector de acción social en España. Situación y retos en un contexto de crisis", en Revista Tercer Sector, núm. 23. Disponible en: http://www.fundacionluisvives.org/rets/23/articulos/101406/index.html $\quad[16$ de febrero de 2014].

Piat Myra y Judith Sabetti (2010), "Residential Housing for Persons with Serious Mental Illness: The Fifty Year Experience with Foster Homes in Canada”, en Stone, J. H. y M. Blouin [eds.], International Encyclopedia of Rehabilitation, Disponible en: http:// cirrie.buffalo.edu/encyclopedia/en/article/236/ [12 de enero de 2013].

Pleace, Nicholas y Allison Wallace (2011), Demonstrating the Effectiveness of Housing Support Services for People with Mental Health Problems: A Review, Reino Unido: National Mental Health Development Unit.

Pleace, Nicholas y Deborah Quilgars (2013), Improving Health and Social Integration through Housing First: A Review, Reino Unido: Centre for Housing Policy.

Prieto-Rodríguez, Adriana (2002), "Salud Mental: situación y tendencias", en Revista de Salud Pública, vol. 4, núm. 1, Colombia: Scielo.

Ridgway, Priscilla (2008), "Supported Housing”, en Mueser, Kim T. y Dilip V. Jester [eds.], Clinical handbook of schizophrenia, Nueva York: The Gilford Press.

Ridgway, Priscilla y Anthony Zipple (1990), “The paradigm shift in residential services: from the linear continuum to supported housing approaches”, en Psychosocial Rehabilitation Journal, vol. 13, núm. 4, USA: American Psychological Association.

Rodríguez-González, Abelardo [coord.] (2002), Rehabilitación Psicosocial de Personas con Trastornos Mentales Crónicos, Madrid: Ediciones Pirámide.

Rogers, Sally et al. (2009), Systematic Review of Supported Housing Literature, 1993-2008, Boston: Boston University, Center for Psychiatric Rehabilitation.

Román-Brugnoli et al. (2014), "Solidaridad en el debate global y local: reflexión desde un análisis del caso chileno", en Convergencia Revista de Ciencias Sociales, núm. 66, México: Universidad Autónoma del Estado de México.

Rosendal, Niels Jensen (2013), "Social Pedagogy in modern times", en Education Policy Analysis Archives, vol. 21, núm. 36. Disponible en: http://epaa.asu.edu/ojs/article/ view/1217/ [3 de mayo de 2013].

Ruggeri, Mirella et al. (2000), "Definition and prevalence of severe and persistent mental illness”, en British Journal of Psychiatry, núm. 177, Reino Unido: The Royal College of Psychiatrists.

Scheyett et al. (2013), "Recovery in severe mental illnesses: a literature review of recovery measures”, en Social Work Research, vol. 37, núm. 3, Washington: National Association of Social Workers.

Segal, Steven P. y Pamela L. Kotler (1993), “Sheltered care residence: ten-year personal outcomes”, en American Journal of Orthopsychiatry, vol. 63, núm. 1, USA: Wiley. 
Siegel, Carol E. et al. (2006), "Tenant outcomes in supported housing and community residences in New York City”, en Psychiatric Services, vol. 57, núm. 7, USA: American Psychological Association.

Stanhope, Victoria y Kerry Dunn (2011), "The curious case of Housing First: The limits of evidence based policy”, en International Journal of Law Psychiatry, vol. 34, núm. 4, Ámsterdam: Elsevier.

Tsemberis, Sam (2010), "Housing First: ending homelessness, promoting recovery and reducing costs", en Ellen, Ingrid Gould y Brian O'Flaherty [eds.], How to House the Homeless, Nueva York: Russell Sage Foundation.

Tsemberis, Sam y Ronda Eisenberg (2000), "Pathways to housing: supported housing for street-dwelling homeless individuals", en Psychiatric Services, vol. 51, núm. 4, USA.

Úcar, Xavier (2013), "Exploring different perspectives of Social Pedagogy: towards a complex and integrated approach", en Education Policy Analysis Archives, vol. 21, núm. 36. Disponible en: http://epaa.asu.edu/ojs/article/view/1282 [3 de mayo de 2013].

Vásquez-Rocca, Adolfo (2011), "Antipsiquiatría. Deconstrucción del concepto de enfermedad mental y crítica de la razón psiquiátrica”, en Nómadas. Revista Crítica de Ciencias Sociales y Jurídicas, vol. 31, núm. 3, Madrid: Universidad Complutense de Madrid

Whitley, Rob y Rosalyn Denise Campbell (2014), "Stigma, agency and recovery amongst people with severe mental illness", en Social Sciences \& Medicine, núm. 107, Ámsterdam: Elsevier.

Wong, Yin-Ling et al. (2011), "Social Integration of People with Serious Mental Illness: Network Transactions and Satisfaction", en Journal of Behavioral Health Services \& Research, vol. 38, núm. 1, USA: Springer. 


\section{Anexo}

\section{Tabla 1}

Principales problemáticas de las personas con trastorno mental severo

\section{PRINCIPALES PROBLEMÁTICAS}

Alteraciones provocadas por la enfermedad

Alteraciones del pensamiento

Alteraciones del estado de ánimo

Alteraciones de la sensopercepción

Alteraciones neurocognitivas

Alteraciones de la conducta y del control de impulsos

Dificultades o restricciones para la actividad básica

Autocuidados

Autonomía personal

Control de la conducta

Capacidad para tener iniciativas y motivación

Dificultades o restricciones para la participación social

Relaciones interpersonales pobres y escasas

Problemas para el acceso a servicios sanitarios, sociales y de atención al ciudadano

Dificultades para gestionar su tiempo libre y disfrutar

Participación asociativa, política, actividad para la defensa de sus derechos

Factores ambientales (estigma, accesibilidad, pertenencia grupos sociales, normas sociales, entorno, oportunidades, etcétera)

Factores personales (género, edad, otras enfermedades, personalidad, educación, condición social)

Fuente: Elaboración propia. 
Omar García-Pérez, José Vicente Peña-Calvo y Susana Torío-López. Atención socioeducativa y trastorno mental severo: la vivienda como base de intervención

\section{Tabla 2}

\section{Principales necesidades de las personas con TMS}

\section{NECESIDADES}

Acceso a los servicios básicos (servicios y prestaciones sociales, educación, sanidad, justicia, en una red básica de servicios comunitarios)

Tratamiento adecuado y continuidad de cuidados

Técnicas activas de recuperación (habilidades sociales, actividades de la vida diaria, resolución de conflictos, afrontamiento de síntomas, etcétera)

Apoyo económico

Apoyo a la integración social

Alojamiento y atención residencial

Actividades de ocio y tiempo libre

Integración laboral (formación e inserción en el mundo del trabajo: protegido, con apoyo o normalizado)

Defensa de sus derechos como Grupo vulnerable a desprotección

Apoyo a las familias, red básica de apoyo

Fuente: Elaboración propia. 


\section{Tabla 3}

Diferencias entre el modelo "continuum residencial" y el modelo "supported housing".

\begin{tabular}{ll}
\hline $\begin{array}{l}\text { MODELO DE CONTINUUM } \\
\text { RESIDENCIAL }\end{array}$ & MODELO SUPPORTED HOUSING \\
\hline Contexto residencial de tratamiento & Hogar \\
\hline Indicación profesional & Elección \\
\hline Rol de cliente o usuario & Roles normales como ciudadanos \\
\hline Control por el personal & Control del hogar por el cliente \\
\hline Agrupación por discapacidad & Integración social \\
\hline Contextos preparatorios y de transición & $\begin{array}{l}\text { Aprendizaje en contextos reales } \\
\text { permanentes }\end{array}$ \\
\hline Niveles de servicio estandarizados & $\begin{array}{l}\text { Servicios y apoyos flexibles e } \\
\text { individualizados }\end{array}$ \\
\hline $\begin{array}{l}\text { Entorno con las menores restricciones } \\
\text { posibles }\end{array}$ & Entorno lo más facilitador posible \\
\hline Independencia & Apoyos flexibles de larga duración \\
\hline
\end{tabular}

Fuente: López Álvarez et al. (2004: 108).

Omar García Pérez. Doctor en Pedagogía y Máster en Intervención e Investigación Socioeducativa. Profesor Ayudante Doctor de la Universidad de Oviedo en el Área de Teoría e Historia de la Educación. Es miembro del Grupo de Análisis Sociológico y Cultural de los Procesos Escolares y Educativos. Líneas de investigación: pedagogía y educación social, centrada en el ámbito de las personas con diversidad funcional y la importancia de la acción pedagógica en los programas de apoyo social para personas con trastorno mental; y aspectos relacionados con el binomio "familia-educación" y el ejercicio de corresponsabilidad familiar y educación parental. Publicaciones recientes: Rodríguez-Menéndez, Carmen, José Vicente Peña-Calvo y Omar García-Pérez, "Estudio cualitativo de las diferencias de género en la elección de opciones académicas en los estudiantes del bachillerato científico-técnico", en Teoría de la Educación. Revista Interuniversitaria, vol. 28, núm. 1, España: Universidad de Salamanca (2016); Fernández-García, Carmen-María, Omar García-Pérez y Sara Rodríguez-Pérez, "Los padres y madres ante la toma de decisiones académicas de los adolescentes en la educación secundaria: un estudio cualitativo", en Revista Mexicana de Investigación Educativa, vol. XXI, núm. 71, México: 
Consejo Mexicano de Investigación Educativa (2016); García-Pérez, Omar y Susana Torío-López, "Funcionamiento básico y social de los usuarios de las viviendas supervisadas para personas con trastorno mental severo en Asturias: necesidad de una intervención pedagógica”, en Revista Complutense de Educación, vol. 25, núm. 2, España: Universidad Complutense de Madrid (2014).

José Vicente Peña Calvo. Doctor en Pedagogía por la Universidad de Oviedo. Actualmente ocupa el puesto de Catedrático de Universidad en el área de Teoría e Historia de la Educación de la Universidad de Oviedo. Investigador responsable del Grupo de Investigación de Análisis Sociológico y Cultural de los Procesos Escolares y Educativos. Líneas de investigación: educación y género, y educación parental. Publicaciones recientes: Peña-Calvo, José-Vicente $e$ al., "Perceived Supports and Barriers for Career Development among Second-Year STEM Students", en Journal of Engineering Education, vol. 105, núm. 2, USA: Wiley (2016); Rodríguez-Menéndez, Carmen, Mercedes Inda Caro y José-Vicente Peña-Calvo, "Spanish high school students' interests in technology. Applying social cognitive career theory", en Journal of Career Development, doi: 10.1177/0894845315599253 (2016); Rodríguez-Menéndez, Carmen, Mercedes Inda Caro y José-Vicente Peña-Calvo, "Validación de la teoría cognitivo social de desarrollo de la carrera con una muestra de estudiantes de ingeniería", en Educación XX1, vol. 18, núm. 2, España: Universidad Nacional de Educación a Distancia (2015).

Susana Torío López. Doctora por la Universidad de Oviedo. Profesora Titular de la Universidad de Oviedo en el Área de Teoría e Historia de la Educación en el Departamento de Ciencias de la Educación. Miembro del Grupo de Análisis Sociológico y Cultural de los Procesos Escolares y Educativos. Líneas de investigación: educación parental y la intervención socioeducativa en familia normalizada y en situación de riesgo; pedagogía social y educación permanente. Publicaciones recientes: Torío-López, Susana et al. "Evaluación de un programa experiencial de educación parental: la perspectiva de los educadores como agentes de cambio", en Aula Abierta, núm. 44, España: Elsevier (2016); Torío-López, Susana et al., "Evaluation of de Building Everyday Life positive parenting programme”, en Journal of Children's Services, vol. 10, núm. 2, Irlanda: Emerald (2015); Torío-López, Susana et al., Construir lo cotidiano: programa de educación parental, España: Octaedro (2013).

Recepción: 16 de julio de 2015.

Aprobación: 27 de mayo de 2016. 
